\title{
EDITORIAL
}

\section{Microbial networking in cancer: when two toxins collide}

A recent study by Dejea et al. has demonstrated that two enterotoxigenic bacteria frequently associated with sporadic colorectal cancer, Bacteroides fragilis and pks+Escherichia coli, are found together in biofilms on tissue from patients with familial adenomatous polyposis. In preclinical mouse models, these two bacteria and their corresponding toxins work synergistically to promote colon cancer.

British Journal of Cancer (2018) 118:1407-1409; https://doi.org/10.1038/s41416-018-0101-2

\section{MAIN}

Bacteria colonise virtually all human body surfaces, and their immunomodulatory and metabolic activities are thought to profoundly affect health. Among the various ecological niches within the body, the gastrointestinal tract is home to the most complex microbial ecosystem with the greatest abundance of microorganisms. The intestinal microbiota can be found as luminal planktonic organisms or associated with the mucosal epithelium, sometimes forming biofilm structures. Biofilms formed of polymicrobial communities have been implicated in human diseases such as medical implant infections, cystic fibrosis pneumonia and periodontitis, ${ }^{1}$ and a new study suggests that polymicrobial biofilms may also have a role in hereditary colon cancer. ${ }^{2}$

A number of studies have demonstrated compositional alterations in the luminal or tissue-associated bacteria between healthy and colorectal cancer (CRC) patients. ${ }^{3}$ Among the various microorganisms contributing to the differences in bacterial composition, Enterobacteriaceae family members that carry the genotoxic pks gene island, enterotoxigenic Bacteroides fragilis (ETBF) with the B. fragilis toxin (bft) gene, and Fusobacterium nucleatum have often been reported. ${ }^{4}$ However, the potential interactions between biofilm structures and cancer have attracted less attention. In 2014, a study reported that biofilms were associated with $89 \%$ of right-sided/proximal CRCs versus $13 \%$ of left-sided/distal CRCs, suggesting a correlation between biofilm organisation and carcinogenesis, ${ }^{5}$ although it is still unclear why biofilms appear to be more strongly associated with proximally located sporadic CRCs. In that study, and a follow-up with additional patients, the biofilm structures were characterised by the presence of Enterobacteriaceae, Bacteroidetes and Fusobacterium among others. ${ }^{5,6}$ These findings suggested that interactions between bacteria within biofilms may contribute to the development of CRC.

In support of this idea, the recent study by Dejea et al. $^{2}$ has greatly advanced our understanding of the impact of bacterial interactions in CRC, by showing a synergistic carcinogenic effect in mice between the Enterobacteriaceae pks+Escherichia coli and ETBF. The authors set out to investigate microbial factors that may contribute to disease onset in patients with a hereditary form of CRC; familial adenomatous polyposis (FAP). Using fluorescence in situ hybridisation to detect bacteria, the authors examined biofilm presence in surgical resections from five patients with FAP. Similar to the biofilms identified from patients with sporadic $\mathrm{CRC}^{5}$, the FAP patient biofilms were polymicrobial, invaded the colon mucus and were found on both polyp and normal mucosal tissues. However, in contrast to biofilms from patients with sporadic CRC,
FAP patient biofilms were found throughout the colon (as opposed to primarily in the proximal colon), and were mainly composed of $E$. coli and $B$. fragilis. Previous studies have indicated that colibactin, which is produced from the pks gene island, and bft toxins both promote CRC in animal models. ${ }^{7,8}$ Therefore, the authors investigated the presence of these two genes in cultured bacteria obtained from the mucosal tissues of 25 FAP and 23 normal patients. PCR analysis revealed an increased prevalence of $p k s$ and bft toxin genes in the bacteria from FAP patients versus control patients.

The co-occurrence of ETBF and pks+E. coli was also confirmed within the biofilms from FAP patients, using laser capture microdissection. To examine the combined impact of both bacteria on disease onset, the authors co-colonised two mouse models with ETBF and pks+E. coli: $A p c^{\text {Min } \Delta 716 /+}$ mice (to model FAP) and azoxymethane (AOM)-injected wildtype (WT) mice. Cocolonised AOM/WT mice developed more tumours than AOM/WT mice colonised by either bacterium alone; a phenotype that depended on the presence of both pks and bft. The co-colonised $A p c^{\text {Min } \Delta 716 /+}$ mice had a shortened survival time versus the monocolonised mice (8 weeks versus 15 weeks), although overall tumour numbers were similar between co- and ETBF-colonised mice.

Fecal IgA against specific bacteria have been shown to associate with intestinal pathologies such as inflammatory bowel disease. ${ }^{9}$ Interestingly, Dejea et al. found co-colonised AOM/WT mice displayed an increased fecal IgA response to $E$. coli compared with mono-colonised mice, which correlated with increased $p k s+E$. coli colonisation of the distal colon mucosal tissue. These pieces of evidence suggest that the enhanced tumourigenesis upon co-colonisation related to a shift in the colonisation location of $p k s+E$. coli from the lumen to the mucosal surface, due to the subsequent increase in $E$. coli-specific IgA. In vitro experiments with mucin-producing intestinal epithelial cell monolayers demonstrated that colonisation with $\mathrm{ETBF}$ or $\mathrm{ETBF} / p k s+E$. coli reduced the mucus layer depth to a comparable amount as the mucindegrader Akkermansia muciniphila, thus suggesting that the mucin-degrading ability of ETBF may facilitate $E$. coli relocalisation to the mucosal tissue, by reducing the hindrance caused by the mucus. To test the interplay between mucin-degrading bacteria and genotoxic pks+E. coli, the authors co-colonised AOM/WT mice with $A$. muciniphila and pks+E. coli. Surprisingly, the presence of $A$. muciniphila reduced tumourigenesis compared with pks+E. coli colonisation, highlighting the key role of ETBF in carcinogenesis and demonstrating that altering the mucosal mucin integrity is not sufficient to promote the carcinogenic 


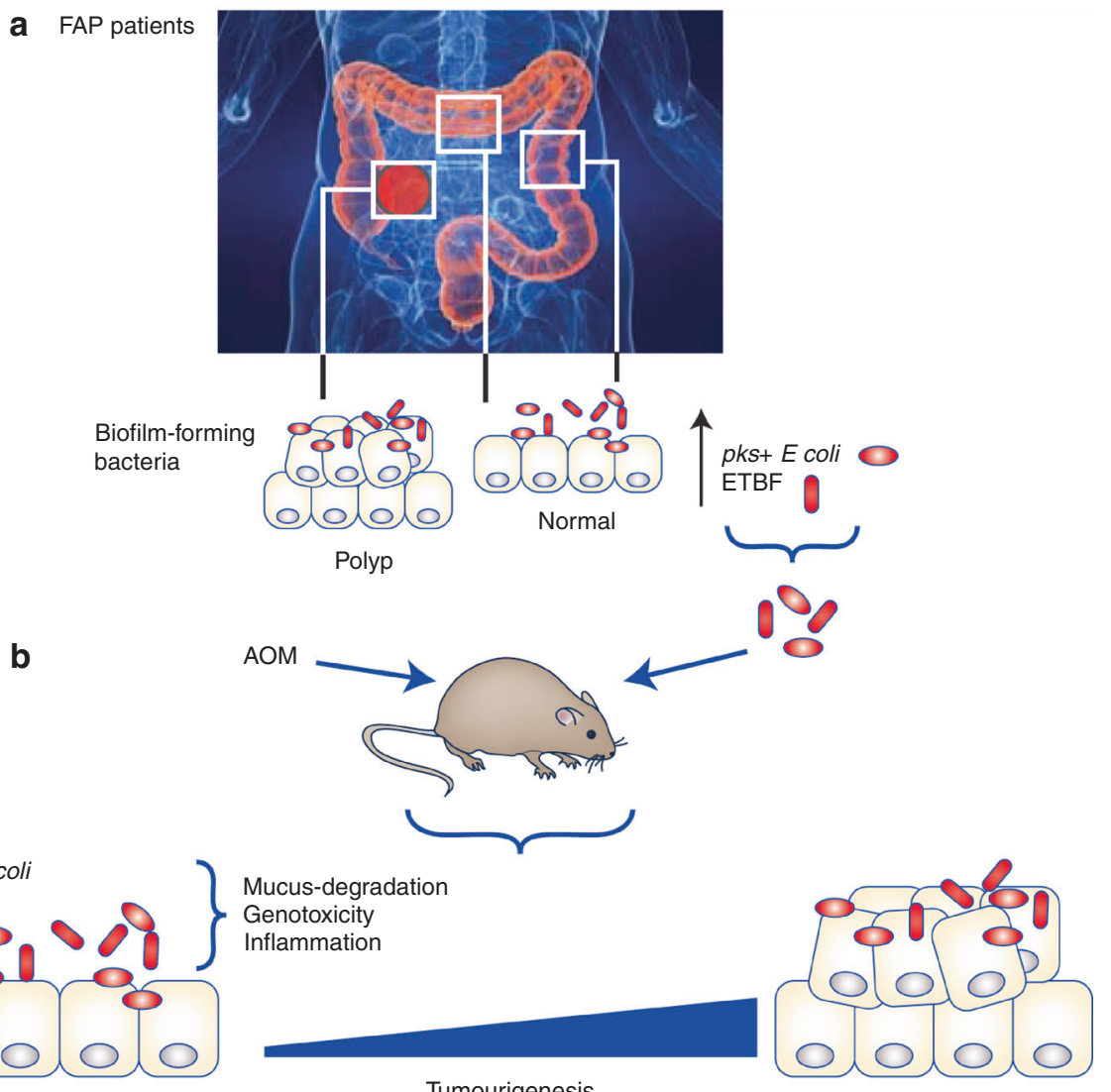

Tumourigenesis

Fig. 1 ETBF and pks+E. coli are found together within FAP patients and synergise to promote colorectal cancer. a Increased prevalence of ETBF carrying Bacteroides fragilis toxin and $E$. coli carrying pks+ toxin, in polyps and macroscopically normal biofilm tissues from FAP patients. b Wildtype mice treated with azoxymethane and co-colonised with ETBF and $p k s+E$. coli developed more tumours than mono-colonised mice. $\mathrm{ETBF}$, through mucin degradation, favours $p k s+E$. coli mucosal tissue colonisation and facilitate pks+-induced genotoxicity. AOM, azoxymethane; FAP, familial adenomatous polyposis; ETBF, enterotoxigenic Bacteroides fragilis

potential of $p k s+E$. coli. These results indicate that additional contributing factors, such as bft, are required for carcinogenesis. Overall, Dejea et al. demonstrated a synergistic interaction between two carcinogenic bacteria, and established the concept of microbial networks in carcinogenesis.

The authors' findings suggest that the spatial distribution of bacteria within the colon, including their proximity to the host mucosal barrier as well as interactions with each other, should be investigated further in the context of CRC. Intestinal lumen and even oral microbial composition has previously distinguished healthy subjects from patients with $\mathrm{CRC}^{10}$; however, these studies were conducted to identify biomarkers, and did not address causation. The evidence that ETBF and E. coli are found within biofilms and mucosal tissue from patients with FAP, and have a combined synergistic effect in animal models of CRC (Fig. 1), suggest that screening for the presence of these two bacterial strains may help assess cancer risk in humans.

The events leading to increased microbial co-occurrence and development of CRC are unclear. One contributing factor appears to be host genetics, as FAP is driven by mutations in the tumoursuppressing adenomatous polyposis coli gene, and $\mathrm{Apc}^{\mathrm{Min} /+}$ mouse models have increased Bacteroides and Enterobacteriaceae compared with WT mice. ${ }^{2}$ B. fragilis and Enterobacteriaceae members have also been identified within mucosal biofilms of intestinal biopsies collected from patients with inflammatory bowel disease, ${ }^{11}$ suggesting intestinal inflammation also influences host susceptibility. In mice, increased mucus penetrability and proximity of bacteria to the mucosal layer are side effects of both high-fat ${ }^{12}$ and western-style ${ }^{13}$ diets, which are both carcinogenic risk factors. Whether diets have similar effects within hereditary CRC patients, by predisposing them to bacterial colonisation of the mucosal surface, is unclear. Interestingly, administering the dietary fibre inulin was shown to ameliorate the negative impact that high-fat or western style diets have on the mucus barrier and bacteria localisation in mice. ${ }^{12,13}$ This may indicate a potential preventative approach.

The polymicrobial nature of CRC suggests additional microbial networking likely exists within the intestine, which could positively or negatively influence carcinogenesis outcomes. It would be important to characterise these microbial networks and identify specific nodes that could represent preventive or therapeutic targets. Dejea et al. provide invaluable information on the functional interaction between two carcinogenic microorganisms, and the study paves the way for future studies elucidating microbial networks in cancer.

\section{ACKNOWLEDGEMENTS}

This research was supported by National Institutes of Health grants R01 DK73338 and from the University of Florida, Department of Medicine Gatorade Fund to C. Jobin.

\section{ADDITIONAL INFORMATION}

Competing interests: The authors declare no competing interests.

Note: This work is published under the standard license to publish agreement. After 12 months the work will become freely available and the license terms will switch to a Creative Commons Attribution 4.0 International (CC BY 4.0). 
Publisher's note: Springer Nature remains neutral with regard to jurisdictional claims in published maps and institutional affiliations.

Sarah Tomkovich ${ }^{1}$ and Christian Jobin ${ }^{1,2,3}$ ${ }^{1}$ Department of Medicine, University of Florida, Gainesville, FL 32611, USA; ${ }^{2}$ Department of Infectious Diseases and Immunology, University of Florida, Gainesville, FL 32611, USA and ${ }^{3}$ Department of Anatomy and Cell Biology, University of Florida, Gainesville, FL 32611, USA Correspondence: Christian Jobin (christian.jobin@medicine.ufl.edu)

\section{REFERENCES}

1. Hall-Stoodley, L., Costerton, J. W. \& Stoodley, P. Bacterial biofilms: from the natural environment to infectious diseases. Nat. Rev. Microbiol. 2, 95-108 (2004).

2. Dejea, C. M. et al. Patients with familial adenomatous polyposis harbor colonic biofilms containing tumorigenic bacteria. Science 359, 592-597 (2018).

3. Borges-Canha, M., Portela-Cidade, J. P., Dinis-Ribeiro, M., Leite-Moreira, A. F. \& Pimentel-Nunes, P. Role of colonic microbiota in colorectal carcinogenesis: a systematic review. Rev. Esp. Enferm. Dig. 107, 659-671 (2015 Nov).

4. Gagnaire, A., Nadel, B., Raoult, D., Neefjes, J. \& Gorvel, J.-P. Collateral damage: insights into bacterial mechanisms that predispose host cells to cancer. Nat. Rev. Microbiol. 15, 109-128 (2017).
5. Dejea, C. M. et al. Microbiota organization is a distinct feature of proximal colorectal cancers. Proc. Natl. Acad. Sci. USA 111, 18321-18326 (2014).

6. Drewes, J. L. et al. High-resolution bacterial $16 \mathrm{~S}$ rRNA gene profile meta-analysis and biofilm status reveal common colorectal cancer consortia. NPJ Biofilms Microbiomes 3, 34 (2017).

7. Arthur, J. C. et al. Intestinal inflammation targets cancer-inducing activity of the microbiota. Science 338, 120-123 (2012).

8. $\mathrm{Wu}, \mathrm{S}$. et al. A human colonic commensal promotes colon tumorigenesis via activation of T helper type $17 \mathrm{~T}$ cell responses. Nat. Med. 15, 1016-1022 (2009).

9. Palm, N. W. et al. Immunoglobulin A coating identifies colitogenic bacteria in inflammatory bowel disease. Cell 158, 1000-1010 (2014).

10. Flemer, B. et al. The oral microbiota in colorectal cancer is distinctive and predictive. Gut pii: gutjnl-2017-314814 (2017).

11. Swidsinski, A., Weber, J., Loening-Baucke, V., Hale, L. P. \& Lochs, H. Spatial organization and composition of the mucosal flora in patients with inflammatory bowel disease. J. Clin. Microbiol. 43, 3380-3389 (2005).

12. Zou, J. et al. Fiber-mediated nourishment of gut microbiota protects against dietinduced obesity by restoring IL-22-mediated colonic health. Cell Host Microbe 23, 41-53.e4 (2018).

13. Schroeder, B. O. et al. Bifidobacteria or fiber protects against diet-induced microbiota-mediated colonic mucus deterioration. Cell Host Microbe 23, 27-40.e7 (2018). 